Über den klaren Gegensatz zwischen O- und R-Ländern hinaus werden in geringerem Umfang auch Initiativen von Ländergruppen gestartet, deren Regierungsparteien sich im Bund als Regierung und Opposition und somit als Kontrahenten gegenüberstehen. Dass diese überparteilichen Initiativen überhaupt zustande kommen und ihre hohe Erfolgsquote von 78 Prozent lässt vermuten, dass bei aller Parteipolitik auch in bescheidenem Maße sachpolitische Länderinteressen in die Gesetzesvorlagen des Bundesrates Eingang finden. In diesem Sinne beantwortet unsere Untersuchung die Ausgangsfrage, ähnlich wie andere Analy$\operatorname{sen}^{32}$, mit einem vorsichtigen „sowohl als auch“. Für eine sicherere und differenziertere Interpretation dieser Ergebnisse sind weitere Studien wünschenswert. ${ }^{33}$

32 Vgl. Thomas Bräuninger/ Thomas Gschwend/Susumu Shikano, a.a.O. (Fn. 8), S. 227.

33 Qualitativ verspricht eine Inhaltsanalyse einzelner Gesetzesvorlagen, insbesondere der M- und Ü-Initiativen näheren Aufschluss. Quantitativ erscheint die getrennte Untersuchung nach Politikfeldern sinnvoll. Burkhart und Manow zeigen beispielsweise, dass die Konfliktintensität bei Finanzen sowie im Politikfeld Arbeit und Soziales besonders hoch ist. Vgl. Simone Burkhart I Philip Manow, a.a.O. (Fn. 22).

\title{
Komplexe Koalitionen: Welchen Nutzen bringen sie den Parteien?
}

\section{Stephan Klecha}

Mit der Etablierung des „fluiden Fünfparteiensystems“1 in Ost- und Westdeutschland sind Regierungsbildungen komplexer geworden. Mehrheiten von einer oder zwei Parteien im Anschluss an Wahlen sind keineswegs mehr sicher. Spätestens die Bundestagswahl 2005 und die Landtagswahlen in Hessen, im Saarland, in Thüringen sowie zuletzt in NordrheinWestfalen haben deutlich gemacht, dass Mehrheiten abseits Großer Koalitionen vielfach nur auf Basis komplexer Koalitionen möglich sind. Darunter werden im Folgenden alle Bündnisse verstanden, in denen mehr als zwei miteinander in Wahlen konkurrierende Parteien auf legislativer oder exekutiver Ebene mit Blick auf die Erlangung von Regierungsmacht oder die Durchsetzung von Regierungshandeln zusammenwirken. Diese Begriffsbestimmung umfasst somit alle formalen Koalitionen sowie die Fälle tolerierter Minderheitsregierungen, die sich im Parlament auf mindestens drei Fraktionen stützen. Mangels Konkurrenz bei den Wahlen zueinander und wegen der Fraktionsgemeinschaft im Deutschen Bundestag werden CDU und CSU hierbei als eine Partei verstanden.

1 Oskar Niedermayer, Nach der Vereinigung: Der Trend zum fluiden Fünfparteiensystem, in: Oscar W. Gabriel / ders. / Richard Stöss (Hrsg.), Parteiendemokratie in Deutschland, Bonn 2001, S. 107 -127 . 


\section{Zu den Schwierigkeiten komplexer Koalitionen}

Wie die jüngsten Erfahrungen zeigen, fällt es den Parteien schwer, komplexe Koalitionen abzuschließen. Den formalisierten Ansätzen zur Koalitionsbildung zufolge ${ }^{2}$ müsste es aber eigentlich relativ einfach sein, diese zu bilden, wenn absolute Mehrheiten fehlen und Mehrheiten aus zwei Parteien (außer der Großen Koalition) ausscheiden. Solchen Bündnissen müssten die Parteien diesen Modellen folgend sogar stets den Vorzug vor Großen Koalitionen geben, nicht zuletzt weil sie möglichst viele Regierungsämter anstreben (office-seeking).

Dennoch zieren sich gerade die kleineren Parteien, überhaupt in Verhandlungen einzutreten. Gespräche zum Abschluss einer Ampelkoalition in Berlin 2001 scheiterten unter anderem am wechselseitigen Misstrauen von Grünen und FDP zueinander. Die FDP verweigerte sich im Bund 2005, in Hessen 2008 und in Nordrhein-Westfalen 2010 energisch allen Avancen, in einer Ampelkoalition mitzuwirken. Die Grünen sondierten nur sehr kurzzeitig und ohne wirkliches Interesse 2005 den Abschluss einer Jamaika-Koalition im Bund und erteilten solchen Optionen dann dezidiert im Bundestagswahlkampf 2009 und im Wahlkampf in Nordrhein-Westfalen 2010 eine Absage. Die Linke schloss ein rot-rotgrünes Bündnis in Nordrhein-Westfalen 2010 nicht aus, war aber anscheinend schon in den Sondierungen nicht bereit, die für ein komplexes exekutives Bündnis besonders wichtige Kompromissbereitschaft einzubringen.

Möglicherweise wirkt das zweite Ziel von Parteien in Koalitionen, Politikinhalte durchzusetzen (policy-seeking), dem entgegen. Die ideologische Nähe der beteiligten Parteien wird als weitere notwendige Voraussetzung für den endgültigen Abschluss einer Koalition an-gesehen ${ }^{3}$. Nimmt man exemplarisch die Untersuchungen von Franz Urban Pappi und Susumu Shikano ${ }^{4}$, so sind die ideologischen Abstände der Parteien zueinander in einer rotrot-grünen oder einer Ampelkoalition größer als in einer Großen Koalition. Auch der Abschluss einer Jamaika-Koalition liegt eher außerhalb des Möglichkeitsraumes. Indes gab es bei der Bundestagswahl 2009, anders als 2005, auf der gesellschaftspolitischen Dimension durchaus eine beträchtliche Nähe von SPD, Grünen und FDP, weniger jedoch in wirtschaftspolitischer Hinsicht ${ }^{5}$. Hierauf stützte insbesondere die SPD ihre Hoffnung, eine Alternative zur Großen Koalition zu besitzen. Dennoch schloss die FDP diese Variante überaus nachdrücklich aus. Tatsächlich ist unter inhaltlichen Gesichtspunkten der Abschluss

2 Vgl. William Gamson, A Theory of Coalition Formation, in: American Sociological Review, 26. Jg. (1961), H. 3, S. 373 - 382; William H. Riker, A Theory of Political Coalitions, New Haven 1962; Michael Leiserson, Factions and Coalitions in One-Party Japan: An Interpretation Based on the Theory of Games, in: American Political Review, 62. Jg. (1968), H. 3, S. 770 - 787; Abram De Swaan, Coalition Theories and Cabinet Formations, Amsterdam 1973; Robert Axelrod, Conflict of Interest: A Theory of Divergent Goals with Applications to Politics, Chicago 1970; Thomas Bräuninger / Marc Debus, Der Einfluss von Koalitionsaussagen, programmatischen Standpunkten und der Bundespolitik auf die Regierungsbildung in den deutschen Ländern, in: PVS, 49. Jg. (2008) H. 2, S. $309-338$.

3 Grundlegend Abram De Swaan, Coalition Theories and Cabinet Formations, a.a.O. (Fn. 2); des Weiteren Robert Axelrod, a.a.O. (Fn. 2).

4 Vgl. Franz Urban Pappi / Susumu Shikano, Regierungsabwahl ohne Regierungsneuwahl, in: PVS, 46. Jg. (2005), H. 4, S. 513 - 526; Franz Urban Pappi, Regierungsbildung im deutschen FünfParteiensystem, in: PVS, 50. Jg. (2009), H. 2, S. $187-202$.

5 Vgl. ebenda, S. 196. 
einer komplexen Koalition sehr schwierig, weil bei solchen Bündnissen ein immenses Konfliktpotential besteht.

Alle Parteien sind vor diesem Hintergrund bemüht, im Vorfeld der Wahlen Signale auszusenden und, dabei besonders unangenehme Konstellationen auszuschließen ${ }^{6}$. Schon mit diesen Koalitionsaussagen binden sie sich äußerst stark an Versprechen, deren Bruch in besonderem Maße Glaubwürdigkeit kostet, wie die hessische SPD 2008 erfahren musste.

Hinzu kommt, dass bestimmte rechnerisch mögliche Koalitionen aus verschiedenen Gründen undenkbar erscheinen. So ist für die Union ein Bündnis mit der Linken oder mit einer rechtsradikalen Partei ausgeschlossen. Die SPD hat in Bezug auf die Linke eine sehr diffizile und nur bedingt konzise Position, die aber vor dem Hintergrund der in der Linken handelnden Personen und ihrer Geschichte beziehungsweise der ihrer Vorläufer PDS und SED leicht nachvollziehbar ist. Koalitionsausschlüsse müssen gleichwohl nicht von Dauer sein. Die Grünen waren in den 1980er Jahren auf Landes- und auf Bundesebene weder für die CDU noch in besonderem Maße für die SPD ein wirklich akzeptabler Partner. Dies hat sich radikal verändert. Die Grünen sind mittlerweile für beide Parteien koalitionsfähig und -willig. Auch das Verhältnis von SPD und Linken ist sehr unterschiedlich und - wie man in Sachsen-Anhalt, Mecklenburg-Vorpommern, Berlin, Brandenburg und teilweise in Thüringen sehen kann - nicht von prinzipieller Abneigung geprägt.

Eine fortwährende Große Koalition kann dabei Alternativen überhaupt erst möglich werden lassen. Ihr Bruch in Berlin 2001 bot dazu ausreichend Anschauungsmaterial, als die damalige PDS zur regierungsfähigen Partei und zum Partner der SPD (und anfangs der Grünen) wurde. Bereits in Niedersachsen lag nach dem Ende einer Großen Koalition 1970 eine Zusammenarbeit der CDU mit einzelnen Vertretern der rechtsradikalen NPD nahe. Vergleichbares lässt sich, etwa am Beispiel der FPÖ/ÖVP-Koalitionen in Österreich, auch international zeigen. Gerade Große Koalitionen können anscheinend zum Inkubator neuer, zuvor undenkbarer Konstellationen werden.

SPD und Union empfinden einander oftmals als fremd, wenn sie ein Bündnis eingehen sollen oder müssen. Deswegen entsteht eine ähnliche Bereitschaft, sich auf zuvor kaum vorstellbare Partner einzulassen, wenn das Verhältnis der jeweiligen Landesverbände von Union und SPD zueinander stark angespannt ist und diese sich besonders schroff voneinander abgrenzen. So resultierte der Abschluss der ersten rot-grünen Koalition in Hessen aus einer solchen besonderen, vor allem regional bedingten Frontstellung der beiden Volksparteien. Weitere Beispiele hierfür sind die rot-grüne Minderheitsregierung mit Duldung der PDS in Sachsen-Anhalt 1994, der Versuch, eine solche in Hessen 2008 zu bilden, oder die Koalition von CDU und FDP mit der rechtspopulistischen Schill-Partei in Hamburg.

Dementsprechend verwundert es nicht, dass SPD und CDU/CSU im Vorfeld der Bundestagswahl 2009 ganz offen Alternativen zur Großen Koalition ins Feld führten, obwohl beide Seiten ihre gemeinsame Arbeit als erfolgreich einstuften. Um in jedem Falle über andere Bündnisoptionen zu verfügen, schlossen sie dabei auch komplexe Koalitionen nicht grundsätzlich aus. Die SPD beschrieb in ihrem Wahlprogramm sogar eine Ampelkoalition explizit als Wunschoption, und tatsächlich ist sie diejenige Partei, die in den Ländern seit 1990 komplexe Koalitionen am häufigsten angestrebt hat, nämlich neun Mal. Auch die

6 Vgl. Frank Decker, Koalitionsaussagen der Parteien vor Wahlen. Eine Forschungsskizze im Kontext des deutschen Regierungssystems, in: ZParl, 40. Jg. (2009), H. 2, S. 431 - 453. 
Union hat mittlerweile Erfahrungen in zwei Versuchen gesammelt beziehungsweise sammelt diese gegenwärtig noch im Saarland.

Dennoch sind komplexe Koalitionen immer noch eher Gedankenspiele als Realität. Möglicherweise ist es nämlich nicht allein die ideologische Distanz und die daraus resultierende Furcht vor schwierigen Verhandlungsprozessen innerhalb einer Koalition, die die Parteien zögern lässt, solche Koalitionen abzuschließen oder sie bewusst anzustreben. Unterstellt man den Parteien, dass sie nicht nur auf die Regierungsübernahme und die Umsetzung einer bestimmten Regierungspolitik zielen, sondern zudem ihren langfristigen Erfolg bei Wahlen in den Blick nehmen (vote-seeking) und dabei auch an einer dauerhaften Mehrheitsfähigkeit arbeiten, so weist der Abschluss einer Koalition stets über die konkrete Wahlperiode hinaus. Dementsprechend zahlt sich der Erfolg oder Misserfolg frühestens bei der darauf folgenden Wahl aus. Diese Erwägungen werden von den Parteien beim Abschluss einer Koalition schon mitbedacht. So werden ihr „Modellcharakter" betont, die zentralen inhaltlichen Ziele als „Projekt“ definiert oder die Zugehörigkeit zum Lager gemeinsamer lebensweltlicher Anschauungen herausgearbeitet. Stets wird dabei der Blick auf die nächsten anstehenden Wahlen gerichtet und abgewogen, wie die Wähler eine mögliche Regierungskonstellation bewerten könnten.

Gerade der Erfolg der bisherigen Bündnisse wäre im Hinblick auf die voraussichtlich bunter werdende Koalitionslandschaft für die Parteien ein essentielles Kriterium, um zu entscheiden, ob sie das durchaus vorhandene Wagnis einer komplexen Koalition eingehen oder nicht.

\section{Vorgehensweise}

An Hand von zwei Kriterien wird der Erfolg einer komplexen Koalition aus Sicht der beteiligten Parteien untersucht: Erstens der Verbleib in der Regierungsverantwortung nach Ende der Koalition, zweitens das Abschneiden bei den folgenden Landtagswahlen. Das Ende einer Koalition wird dabei entweder durch Neuwahlen oder durch eine Veränderung in der Regierungszusammensetzung, etwa durch Sturz des Ministerpräsidenten oder Veränderung bei den Koalitionspartnern, definiert. Nicht berücksichtigt wird der Wechsel im Amt des Ministerpräsidenten (etwa durch Tod oder Rücktritt des Amtsinhabers), sofern die Koalition ansonsten unverändert bleibt. Untersucht wird dabei der Zustand der Partei vor und nach dem Ende der Koalition. Verbleibt eine Partei in der Regierungsverantwortung, so ist der Erfolg der komplexen Koalition positiv, scheidet sie aus, ist er negativ. Falls eine Regierung vorzeitig zerbricht, jedoch bis zu vorgezogenen oder ohnehin anstehenden Neuwahlen im Wesentlichen unverändert im Amte bleibt, so wird als neue Regierung diejenige zugrunde gelegt, die nach den Wahlen gebildet wurde. Für das Abschneiden bei den Landtagswahlen gilt: Steigert eine Partei ihren Stimmenanteil, ist das Ergebnis der Koalition aus ihrer Sicht positiv. Verliert sie Stimmenanteile, ist es negativ.

Bei den vorliegenden Fällen tolerierter Minderheitsregierungen wird die tolerierende Partei der Regierung zugehörig zugeordnet, sofern auf legislativer Ebene faktisch eine privilegierte Kooperation existierte. Die Untersuchung beschränkt sich auf die nach 1949 abgeschlossenen oder konkret angebahnten Koalitionen. Als konkret angebahnt werden diejenigen Bündnisse eingeschlossen, in denen eine komplexe Koalition bereits fest vereinbart war und auch Koalitions- beziehungsweise Tolerierungsvereinbarungen bestanden (wie in 
Schleswig-Holstein 2005 und in Hessen 2008) oder in denen die Sondierungsgespräche zwischen den Parteien bereits so weit fortgeschritten waren, dass die Koalitionsbildung höchstwahrscheinlich stattfinden würde (wie in Thüringen 2009). Weil im Saarland der Abschluss des Jamaika-Bündnisses nur alternierend zum Modell einer rot-rot-grünen Koalition möglich war, wird in diesem Fall lediglich das tatsächlich abgeschlossene Bündnis einbezogen.

\section{Komplexe Koalitionen in der Bundesrepublik}

Seit der Gründung der Bundesrepublik hat es 34 Fälle gegeben, in denen eine komplexe Koalition abgeschlossen worden ist oder konkret angebahnt wurde. Bei rund 200 Wahlen zu Bundes- oder Landtagen seit 1949 und den danach obligatorischen Regierungsneubildungen ist demnach in ungefähr jedem siebten Fall eine komplexe Koalition angestrebt worden beziehungsweise auch ins Amt gelangt. Zwei dieser Bündnisse waren dabei auf Bundesebene zustande gekommen, wobei dies bei der zweiten Regierung unter Konrad Adenauer für die eigentliche Regierungsbildung an sich entbehrlich gewesen wäre, wenn nicht damit die erforderliche Mehrheit für die Verabschiedung der Wehrverfassung erzielt worden wäre. Danach hat es rechnerisch im Bund bis auf 2005 stets für eine kleine Koalition aus Union oder SPD mit einem weiteren Partner (FDP oder Grüne) gereicht, die dann auch immer erfolgreich gebildet wurden.

In den Ländern sind zwei Phasen komplexer Koalitionen zu unterscheiden. In der Gründungsphase der Bundesrepublik Deutschland waren sie sehr häufig anzutreffen. Außer in Hessen existierten solche komplexen Bündnisse in allen Ländern, sofern man den Hamburgblock von 1953 ebenfalls diesem Typus zuordnet. Diese nach außen hin unitarisch auftretende Partei war eigentlich ein Parteienkartell aus CDU, DP und FDP, das sich wegen des damaligen Wahlrechts zur Hamburgischen Bürgerschaft gebildet hatte ${ }^{7}$. Somit kann dieser durchaus den komplexen Koalitionen zugerechnet werden.

Bis zur Mitte der 1960er Jahre korrespondierte die beträchtliche Koalitionsvielfalt einerseits mit der Fragmentierung des bürgerlichen Lagers und andererseits mit der teilweise immer noch anzutreffenden Neigung, übergroße Koalitionen, gar Allparteienkoalitionen, abzuschließen. Solch übergroße Koalitionen waren vor allem in Baden-Württemberg und im Saarland anzutreffen. Beide Bundesländer existierten 1949 noch nicht, sondern wurden erst später gebildet beziehungsweise traten erst später der Bundesrepublik bei. Beide Male war der Zusammenschluss von mehr als zwei Parteien nicht zwingend für die parlamentarische Mehrheitsfähigkeit der Koalition, wohl aber für den Aufbau des Gemeinwesens im Lande beziehungsweise die Integration des Landes in die Bundesrepublik förderlich.

Die Fragmentierung des bürgerlichen Lagers drückte sich darin aus, dass es neben der Union als überkonfessioneller Sammlungsbewegung eine Vielzahl von Parteien gab, die entweder spezifische Anliegen vertraten (BHE), konfessionell im Gegensatz zur CDU/CSU eng gebunden blieben (Zentrum) oder in denen sich regionalistische Interessen bündelten (BP und teilweise DP). Solange diese Kräfte neben der Union wirkten, waren komplexe

7 Vgl. Julia von Blumenthal, Freie und Hansestadt Hamburg: Das letzte Feierabendparlament, in: Siegfried Mielke / Werner Reutter (Hrsg.), Länderparlamentarismus in Deutschland, Geschichte - Struktur - Funktionen, Wiesbaden 2004, S. 195 - 224, S. 201. 


\begin{tabular}{|c|c|c|c|}
\hline \multicolumn{4}{|c|}{ Tabelle 1: Komplexe Koalitionen in der Bundesrepublik Deutschland nach 1949} \\
\hline & Jahr & Koalition & Anmerkung \\
\hline Bund & 1949 & $\mathrm{CDU} / \mathrm{CSU} / \mathrm{FDP} / \mathrm{DP}$ & \\
\hline Schleswig-Holstein & 1950 & $\mathrm{CDU} / \mathrm{BHE} / \mathrm{DP} / \mathrm{FDP}$ & zeitweise CDU / FDP-Minderheitsregierung \\
\hline Berlin & 1951 & $\mathrm{SPD} / \mathrm{CDU} / \mathrm{FDP}$ & $\begin{array}{l}\text { Koalition zerbricht nach Tod des Regierenden } \\
\text { Bürgermeisters Ernst Reuter. }\end{array}$ \\
\hline Bremen & 1951 & SPD / FDP / CDU & \\
\hline Niedersachsen & 1951 & SPD / Z / BHE & \\
\hline Baden-Württemberg & 1952 & FDP / SPD / BHE & \\
\hline Hamburg & 1953 & Hamburgblock & $\begin{array}{l}\text { CDU, FDP und DP sind mit gemeinsamer Liste } \\
\text { zur Wahl angetreten. }\end{array}$ \\
\hline Bund & 1953 & $\begin{array}{l}\mathrm{CDU} / \mathrm{CSU} / \mathrm{FDP} / \\
\mathrm{BHE} / \mathrm{DP}\end{array}$ & $\begin{array}{l}\text { FDP verlässt } 1956 \text { die Koalition, Minister blei- } \\
\text { ben jedoch im Amt und bilden mit einem Teil } \\
\text { der Fraktion die FVP. }\end{array}$ \\
\hline Baden-Württemberg & 1953 & $\mathrm{CDU} / \mathrm{SPD} / \mathrm{FDP} / \mathrm{BHE}$ & \\
\hline Bayern & 1954 & SPD / BP / BHE / FDP & \\
\hline Schleswig-Holstein & 1954 & $\mathrm{CDU} / \mathrm{BHE} / \mathrm{FDP}$ & \\
\hline Nordrhein-Westfalen & 1954 & CDU / Z / FDP & \\
\hline Bremen & 1955 & SPD / FDP / CDU & \\
\hline Niedersachsen & 1955 & $\mathrm{DP} / \mathrm{CDU} / \mathrm{FDP} / \mathrm{BHE}$ & \\
\hline Nordrhein-Westfalen & 1956 & SPD / FDP / Z & per konstruktivem Misstrauensvotum etabliert \\
\hline Baden-Württemberg & 1956 & $\begin{array}{l}\mathrm{CDU} / \mathrm{SPD} / \mathrm{FDP} / \\
\mathrm{BHE}\end{array}$ & $\begin{array}{l}\text { Nach Rücktritt von Ministerpräsident Gebhard } \\
\text { Müller } 1958 \text { Fortführung der Koalition unter } \\
\text { Kurt Georg Kiesinger. }\end{array}$ \\
\hline Saarland & 1957 & CDU / SPD / DPS & \\
\hline Bayern & 1957 & CSU / BHE / FDP & Nach Bruch der Viererkoalition ins Amt gelangt. \\
\hline Niedersachsen & 1957 & $\mathrm{DP} / \mathrm{SPD} / \mathrm{CDU}$ & $\begin{array}{l}\text { Regierungsumbildung, Ministerpräsident Hein- } \\
\text { rich Hellwege bleibt im Amt. }\end{array}$ \\
\hline Bayern & 1958 & CSU / FDP / BHE & $\begin{array}{l}\text { Koalition wird nach Tod von Ministerpräsident } \\
\text { Hanns Seidel unter Hans Ehard fortgeführt. }\end{array}$ \\
\hline Niedersachsen & 1959 & SPD / FDP / BHE & $\begin{array}{l}\text { Wird nach Tod von Ministerpräsident Hinrich } \\
\text { Wilhelm Kopf } 1961 \text { unter Georg Diederichs fort- } \\
\text { geführt. }\end{array}$ \\
\hline Saarland & 1959 & $\mathrm{CDU} / \mathrm{SPD} / \mathrm{CVP}$ & $\begin{array}{l}\text { Nach dem Tod von Ministerpräsident Hans Egon } \\
\text { Reinert scheidet CVP aus. }\end{array}$ \\
\hline Baden-Württemberg & 1960 & $\mathrm{CDU} / \mathrm{FDP} / \mathrm{BHE}$ & \\
\hline Brandenburg & 1990 & SPD / FDP / B90 & \\
\hline Bremen & 1991 & SPD / FDP / Grüne & \\
\hline Sachsen-Anhalt & 1994 & SPD / B90/Grüne & Minderheitsregierung, toleriert von PDS \\
\hline Hamburg & 2001 & CDU / Schill-Partei / FDP & \\
\hline Berlin & 2001 & SPD / B90/Grüne & Minderheitsregierung, toleriert von PDS \\
\hline Berlin & 2001 & SPD / FDP / B90/Grüne & Verhandlungen scheitern \\
\hline Schleswig-Holstein & 2005 & SPD / B90/Grüne & $\begin{array}{l}\text { Minderheitsregierung, toleriert vom SSW; Wahl } \\
\text { der Ministerpräsidentin scheitert }\end{array}$ \\
\hline Hessen & 2008 & SPD / B90/Grüne & $\begin{array}{l}\text { Minderheitsregierung, toleriert von Die Linke; } \\
\text { Wahl der Ministerpräsidentin scheitert }\end{array}$ \\
\hline Saarland & 2009 & CDU / FDP / B90/Grüne & Parallele Gespräche SPD / Linke / Grüne \\
\hline Thüringen & 2009 & $\begin{array}{l}\text { Die Linke / SPD / B90/ } \\
\text { Grüne }\end{array}$ & Verhandlungen scheitern \\
\hline Nordrhein-Westfalen & 2010 & SPD / B90/Grüne & $\begin{array}{l}\text { Minderheitsregierung, de facto toleriert von } \\
\text { Die Linke }\end{array}$ \\
\hline uelle: Eigene Zus & & d Darstellung, vgl. au & $\begin{array}{l}\text { Aufsätze im Sammelband Uwe Jun / Melanie } \\
\text { ysteme in den deutschen Ländern, Wiesbaden }\end{array}$ \\
\hline
\end{tabular}


Koalitionen für eine Mehrheitsfähigkeit der Union oftmals zwingend notwendig, weil gerade die Differenzen im bürgerlichen Lager der SPD Mehrheitsoptionen eröffneten.

Nachdem sich dann in den 1960er Jahren ein "Zweieinhalbparteiensystem "8 herausgebildet hatte, in dem die FDP eine unbestrittene Schlüsselrolle für die Regierungsbildung im Bund wie in den Ländern besaß, bedurfte es keiner komplexen Koalition mehr. Stets hatten maximal zwei Parteien zusammen eine absolute Mehrheit der Mandate. Einzig 1979 hätte es in Schleswig-Holstein eine komplexe Koalition aus SPD, FDP und SSW geben können. Nur das Wahlrecht verhinderte dies knapp, weil die CDU, begünstigt durchs Wahlrecht die absolute Mehrheit der Mandate erlangte.

Auch nach dem Aufkommen der Grünen in den 1980er Jahren waren komplexe Koalitionen nicht zwingend erforderlich. Vielmehr hatten sich zwei Lager herausgebildet, innerhalb derer sich die Mehrheitsbildung vollzog. Die Parteien sahen sich als „Allianz der verschiedenen Lebenswelten lediglich eines Lagers (...), als eine Art von Binnenintegration, nicht als die strategische Möglichkeit, komplementäre soziale und kulturelle Kräfte neu zu bündeln"9. Diese Lager manifestierten sich auf der einen Seite in Koalitionen aus Union und FDP, auf der anderen Seite in SPD/Grüne-Bündnissen. Abweichend davon waren in den Ländern jedoch aus regionalen Spezifika heraus auch Große Koalitionen (etwa in Bremen ab 1995), SPD/FDP-Koalitionen (in den 1980er Jahren in Hamburg, ab 1991 in Rheinland-Pfalz) oder ganz anders gelagerte Regierungsbündnisse (wie SPD/Statt-Partei in Hamburg) gebildet worden. Darin liegt noch kein Bruch mit dem Vorrang von Zweiparteienkoalitionen gegenüber komplexen Bündnissen, obwohl dies nach dem Hinzutreten der PDS als fünfter Kraft nach 1990 zu erwarten gewesen wäre.

Allerdings war das Fünfparteiensystem noch unfertig. In den Ländern hatte eine Regionalisierung des Parteiensystems stattgefunden, bei der komplexe Koalitionen weiterhin sehr unwahrscheinliche Konstellationen blieben. Lediglich auf Bundesebene war die Bildung von Zweiparteienkoalitionen fraglich geworden, noch aber reichten die Mehrheiten für Zweierbündnisse. Abweichend entwickelte sich nur das Land Berlin in den 1990er Jahren, wo phasenweise bereits ein Fünfparteiensystem existierte beziehungsweise die PDS als Koalitionspartner so eindeutig ausgeschlossen war, dass es keine Alternativen zur Großen Koalition gab. Ebenso wichen kurzzeitig Brandenburg 1990 und Baden-Württemberg 1992 ab, als dort ebenfalls fünf Parteien den Einzug in den Landtag schafften und durch den Ausschluss von Republikanern und PDS aus dem Kreis möglicher Koalitionspartner Zweiparteienbündnisse nur in Form Großer Koalitionen möglich waren. Während in Brandenburg dabei auf eine Ampelkoalition ausgewichen wurde, blieb in Baden-Württemberg nur der Abschluss einer Großen Koalition übrig. Zur Ampel in Bremen und zur rot-grünen Minderheitsregierung in Sachsen-Anhalt gab es hingegen vor dem Hintergrund der Kräfteverhältnisse in den Landtagen noch stets Alternativen, eine kleine Koalition aus zwei Parteien zu bilden. Gleichwohl kann festgehalten werden, dass in den 1990er Jahren komplexe Koalitionen die bewährten lagerimmanenten Zweiparteienbündnisse keineswegs ersetzt, allenfalls punktuell ergänzt hatten.

8 Oskar Niedermayer, a.a.O. (Fn. 1), S. 107.

9 Johanna Klatt / Franz Walter, Politik und Gesellschaft am Ende der Zweiten Großen Koalition und was folgt? Konklusion und Ausblick, in: Felix Butzlaff / Stine Harm / Franz Walter (Hrsg.), Patt oder Gezeitenwechsel? Deutschland 2009, Wiesbaden 2009, S. 295 - 322. 
In den 2000er Jahren verändert sich die Koalitionslandschaft. Waren Hamburg und Berlin 2001 ebenso wie Schleswig-Holstein 2005 noch stark unter dem Fokus regionaler Besonderheiten zu vermerken, so ist seit der Bundestagswahl 2005 das Fünfparteiensystem so weit Realität, dass klare Mehrheit für ein gewohntes Bündnis einer kleinen Koalition mit zwei Parteien erheblich erschwert sind. Die faktische Abspaltung eines Teils der SPD-Wählerschicht mit Neugründung der WASG und ihr Zusammengehen mit der PDS zur Partei „Die Linke“ führten seit 2007 auch in westdeutschen Landtagen dazu, dass dort eher fünf als vier Parteien vertreten sind. Nachdem seit 2002 auch FDP und Grüne wieder einigermaßen regelmäßig die Sperrklausel bei Landtagswahlen in Ostdeutschland überwunden haben, hat sich so mittlerweile gesamtdeutsch ein „fluides Fünfparteiensystem“ herausgebil$\operatorname{det}^{10}$, in dem sich die regionalen Stärken der einzelnen Parteien sehr stark unterscheiden und das außerdem noch einige regionale Besonderheiten wie die Freien Wähler in Bayern, den SSW in Schleswig-Holstein oder rechtsextreme Parteien in Ostdeutschland aufweist. Seitdem sind Parlamente mit fünf oder sogar mehr Parteien (gegenwärtig sind sechs Parteien in den Landtagen von Sachsen und Schleswig-Holstein vertreten) die Regel in den Ländern. Komplexe Koalitionen werden zur Regierungsübernahme deswegen öfter erwogen beziehungsweise sind zwingend erforderlich, wenn eine Große Koalition vermieden werden soll. Die Beurteilung komplexer Koalitionen nach ihrem Erfolg ist also eindeutig eine Frage, die langfristig von wachsendem Interesse für die Parteien sein wird.

\subsection{Kriterium Regierungsbeteiligung}

Beim Kriterium Regierungsbeteiligung wird untersucht, ob eine Partei nach dem Ende der komplexen Koalition weiterhin an der Regierung exekutiv und legislativ beteiligt ist.

Zentraler Akteur in den 1950er Jahren war zum einen die FDP, die bis auf zwei Fälle in Niedersachsen an allen komplexen Koalitionen beteiligt war. Damit wird bereits ihre Scharnierfunktion deutlich, die sie in den 1960er Jahren zwischen SPD und der Union einnehmen würde. Zum anderen hatte anfangs aber auch der BHE eine vergleichbare Funktion, die sich vor allem in Bayern und Niedersachsen im Abschluss komplexer Koalitionen ausdrückte, aber auch bei der Bildung kleiner Regierungsbündnisse in Hessen von Bedeutung war. Der BHE war allerdings als Interessenvertretung der Vertriebenen eine Partei, die mit der gesellschaftlichen Integration der Flüchtlinge ihr zentrales Anliegen Stück für Stück verlor und sich deswegen auch im Verlauf der 1960er Jahre auflöste.

Während die Koalitionslandschaft in den 1950er Jahren überaus heterogen war, sind komplexe Koalitionen nach 1990 recht gleichförmig gewesen: Mit Ausnahme des Hamburger Senats von 2001 waren bis 2009 komplexe Koalitionen in der zweiten Phase nämlich stets rot-grüne Bündnisse mit einem weiteren Partner, wobei die SPD ihre Regierungsbeteiligung auch nach dem Ende der Koalition in der Regel sichern konnte, die Grünen hingegen nie.

10 Vgl. Oskar Niedermayer, a.a.O. (Fn. 1); ders., Von der Zweiparteiendominanz zum Pluralismus: Die Entwicklung des deutschen Parteiensystems im westeuropäischen Vergleich, in: PVS, 51. Jg. (2010), H. 1, S. 1 - 13. 


\begin{tabular}{|c|c|c|c|c|c|c|}
\hline & SPD & $\mathrm{CDU} / \mathrm{CSU}$ & FDP & Grüne & Linke & Sonstige \\
\hline Bund 1949 & & ja & ja & & & DP ja \\
\hline SH 1950 & & ja & ja & & & BHE ja, DP nein \\
\hline BE 1951 & nein & ja & ja & & & \\
\hline НB 1951 & ja & ja & ja & & & \\
\hline NDS 1951 & nein & & & & & BHE ja, $Z$ nein \\
\hline BW 1952 & ja & & ja & & & BHE ja \\
\hline НH 1953 & & nein & ja & & & DP nein \\
\hline Bund 1953 & & ja & nein & & & BHE nein, DP ja \\
\hline BW 1953 & ja & ja & ja & & & BHE ja \\
\hline BY 1954 & nein & & ja & & & $\mathrm{BP}$ nein, $\mathrm{BHE}$ ja \\
\hline SH 1954 & ja & & ja & & & BHE nein \\
\hline NRW 1954 & & nein & ja & & & $Z_{j a}$ \\
\hline HB 1955 & ja & nein & ja & & & \\
\hline NDS 1955 & & ja & nein & & & BHE ja \\
\hline NRW 1956 & nein & & nein & & & $Z_{\text {nein }}$ \\
\hline BW 1956 & nein & ja & ja & & & BHE ja \\
\hline SL 1957 & ja & ja & nein & & & \\
\hline BY 1957 & & ja & ja & & & BHE ja \\
\hline NDS 1957 & ja & ja & & & & BHE nein, DP nein \\
\hline BY 1958 & & ja & nein & & & BHE nein \\
\hline NDS 1959 & ja & & ja & & & BHE nein \\
\hline SL 1960 & nein & ja & & & & CVP nein \\
\hline BW 1960 & nein & ja & ja & & & BHE nein \\
\hline BB 1990 & ja & & nein & nein & & \\
\hline НВ 1991 & ja & & nein & nein & & \\
\hline SAN 1994 & ja & & & nein & (ja) & \\
\hline НH 2001 & & ja & nein & & & Schill-Partei nein \\
\hline BE 2001 & ja & & & nein & (ja) & \\
\hline BE 2002 & ja & & nein & nein & & \\
\hline SH 2005 & ja & & & nein & & SSW (nein) \\
\hline HE 2008 & nein & & & nein & (nein) & \\
\hline ТH 2009 & ja & & & nein & nein & \\
\hline \multicolumn{7}{|c|}{$\begin{array}{l}\text { Anmerkung: Ist eine Partei nur legislativ, eben durch Stützung einer Minderheitsregierung beteiligt, so } \\
\text { ist der entsprechende Fall eingeklammert. Die gegenwärtigen Regierungen im Saarland und in Nord- } \\
\text { rhein-Westfalen können nicht unter dem Kriterium späterer Regierungsbeteiligung beurteilt werden. Für } \\
\text { den Hamburgblock ist eine Differenzierung in die Quellparteien vorgenommen worden. } \\
\text { Quelle: Eigene Zusammen- und Darstellung, vgl. auch Aufsätze im Sammelband Uwe Jun / Melanie } \\
\text { Haas / Oskar Niedermayer (Hrsg.), Parteien und Parteiensysteme in den deutschen Ländern, Wiesbaden } \\
\text { 2009. }\end{array}$} \\
\hline
\end{tabular}




\begin{tabular}{|l|c|c|c|c|}
\hline \multirow{2}{*}{ Tabelle 3: Erfolg komplexer Koalitionen nach dem Kriterium Regierungsbeteiligung } \\
\cline { 2 - 5 } & Beteiligt & Erfolgreich & Beteiligt & Erfolgreich \\
\hline SPD & 23 & 15 & 8 & 7 \\
CDU/CSU & 18 & 15 & 1 & 1 \\
FDP & 24 & 15 & 4 & 0 \\
Grüne & 8 & 0 & 8 & 0 \\
PDS/Linke & 4 & 2 & 4 & 2 \\
BHE & 14 & 8 & & \\
DP & 5 & 2 & & 0 \\
Zentrum & 3 & 1 & 1 & 0 \\
Schill-Partei & 1 & 0 & 1 & \\
SSW & 1 & 0 & & \\
\hline Quelle: Eigene Darstellung, siehe auch Tabelle 2.
\end{tabular}

SPD und FDP haben über die gesamte Zeit hinweg die meiste Erfahrung im Abschluss oder in der Anbahnung komplexer Koalitionen. Auch die Erfolgsquoten beider Parteien sind auf den ersten Blick einander ähnlich. In etwa zwei Drittel der Fälle blieben die Parteien nach dem Ende der komplexen Koalition weiterhin in Regierungsverantwortung.

Die Union kommt auf eine geringere Zahl an komplexen Koalitionen, weist dafür jedoch eine beachtliche Erfolgsquote auf. Nur in drei der 18 Fälle verfehlte sie hinterher die Regierungsverantwortung. Lediglich zu 50 Prozent war die PDS erfolgreich, wobei hier zwei Fälle von Tolerierungen gezählt worden sind, die einmal (in Berlin 2001) hinterher sogar zur exekutiven Regierungsbeteiligung geführt haben. Die Grünen sind die einzige Partei, die an mehr als einer komplexen Koalition beteiligt waren und hinterher stets in die Opposition wechseln mussten.

Eine komplexe Koalition hat sich also unter dem Gesichtspunkt einer anschließenden Regierungsbeteiligung über alle Zeiten hinweg für die CDU/CSU zumeist, für SPD und FDP in der Regel, für die PDS/Linke teils-teils und für die Grünen nie ausgezahlt.

Etwas anders fällt das Urteil aus, wenn man nur die nach 1990 abgeschlossenen Bündnisse einbezieht. Die SPD hat mit Ausnahme des gescheiterten Anlaufs in Hessen immer auch nach dem Ende der Koalition weiter regieren können. So nutzte sie in SchleswigHolstein 2005 die Alternative der Großen und in Berlin der rot-roten Koalition. 1995 flüchtete sie sich in Bremen und 2009 in Thüringen in Große Koalitionen. Nach Neuwahlen sicherte sie sich eine absolute Mehrheit in Brandenburg und konnte die Tolerierung einer Alleinregierung in Sachsen-Anhalt erreichen. Der Union ist in ihrem bislang einzig beurteilbaren Fall, in Hamburg 2001, danach eine absolute Mehrheit geglückt. Während SPD und Union recht positiv ihre bisherigen komplexen Koalitionen bewerten können, fällt die Bilanz gerade bei der FDP verheerend aus. Sie ist genauso wie die Grünen nach dem Ende eines solchen Bündnisses immer aus der Regierung ausgeschieden.

Unter dem Gesichtspunkt Regierungsteilhabe haben also sowohl die SPD als auch die Union sehr positive Erfahrungen mit komplexen Koalitionen sammeln können; hingegen haben gerade die potentiellen Partner in einer Ampel- oder Jamaika-Koalition nicht von einer solchen Konstellation profitiert. 


\subsection{Kriterium Wahlergebnis}

Stehen die Mehrheitsverhältnisse im Parlament fest, gibt es zu Beginn einer Wahlperiode meist - rechnerisch und/oder tatsächlich - alternative Optionen für eine Regierungsbildung. Dies verhält sich anders in der Beurteilung einer Koalition durch die Wähler. Eine Partei schöpft ja gerade aus Wahlergebnissen ihre Legitimation für eine bestimmte Politik. Dementsprechend drückt das Wählervotum in der Regel auch Koalitionspräferenzen oder -ablehnungen aus.

Es ist einschränkend zu ergänzen, dass der Zeitpunkt einer Wahl nicht unwesentlich ist. Gerade Landtagswahlen haben oft den Charakter von Testwahlen, bei denen die im Bund regierenden Parteien in der Mitte der Wahlperiode eher Einbußen in der Wählergunst hinnehmen müssen, während die Oppositionsparteien eher auf Zugewinne hoffen dürfen ${ }^{11}$. Dennoch stellt das Abschneiden einer Partei bei Landtagswahlen immer auch ein Zeugnis über ihre Arbeit im Lande aus.

Gegenüber dem Kriterium Regierungsbeteiligung fällt die Erfolgsbilanz einer Partei bei Wahlen nach einer komplexen Koalition sehr eindeutig aus.

So hat die SPD ihre Wahlergebnisse außer in Bremen 1991 und Hessen 2008 nach einer komplexen Koalition stets verbessern können. Die beiden einzigen Verschlechterungen der Union lagen in den 1950er Jahren in Bremen und Baden-Württemberg. Beides waren übergroße Koalitionen, an denen auch die SPD beteiligt war. Demgegenüber hat die CDU in Hamburg 2004 einen triumphalen Stimmenzuwachs verbuchen können. Dies gelang auch der SPD in Brandenburg 1994 und in Berlin 2001. Das Ergebnis in Sachsen-Anhalt 1998 war ebenfalls, obgleich der prozentuale Zugewinn eher gering war, sehr gut. Rund ein Drittel mehr Wähler als bei der vorherigen Wahl votierten damals für die SPD. Nimmt man das hessische Desaster hinzu, wird deutlich, dass die Ressource Ministerpräsident außerordentlich wichtig zu sein scheint. Gelingt es, anders als in Bremen, wo der vorherige Bürgermeister weiter amtierte, durch eine komplexe Koalition einen Wechsel im Amt des Regierungschef und gar die Ablösung einer aufgebrauchten Regierung zu organisieren, so honoriert der Wähler auf lange Sicht die machtpolitischen Finessen, die Tabubrüche und die Ränkespiele der Koalitionäre, die dem Abschluss einer komplexen Koalition in der Regel vorangehen.

Schwieriger ist es für die kleineren Parteien. Deutliche Verlierer komplexer Bündnisse sind durchgängig die beiden Scharnierparteien BHE und FDP. Beide konnten nur in jeweils einem einzigen Fall zulegen. Besonders dramatisch ist die Situation für die FDP nach 1990. Nach jeder Teilhabe an einer komplexen Koalition verfehlten die Liberalen hinterher sogar den neuerlichen Einzug in den Landtag. Aus dieser Erfahrung heraus könnte die Skepsis der FDP verständlich werden, warum gerade sie komplexen Koalitionen sehr reserviert gegenübersteht.

Für die Grünen stellt sich die Situation bei den Wahlen hingegen wesentlich besser dar als beim Kriterium Regierungsteilhabe. So folgte zwar in Brandenburg und Sachsen-Anhalt jeweils der Fall unter die Fünfprozenthürde, während die Verluste in Berlin 2001 nur gering waren. Dem stehen Zuwächse in Bremen und sehr deutliche Gewinne in Hessen 2008 gegenüber. Berücksichtigt man bei den Grünen zudem, dass sie bei den Wahlen, die auf das

11 Vgl. Charlie Jeffrey / Dan Hough, Regional Elections in Multi-Level Systems, in: European Urban and Regional Studies, 10. Jg. (2003), H. 3, S. $199-212$. 


\begin{tabular}{|c|c|c|c|c|c|c|}
\hline & \multicolumn{6}{|c|}{ Parlamentarisch } \\
\hline & SPD & CDU/CSU & FDP & Grüne & Linke & Sonstige \\
\hline Bund 1949 & & ja & nein & & & DP nein \\
\hline SH 1950 & & ja & nein & & & BHE nein, DP nein \\
\hline HB 1951 & ja & ja & nein & & & \\
\hline NDS 1951 & ja & & & & & BHE nein, $\mathrm{Z}$ nein \\
\hline НH 1953 & & $\begin{array}{l}\text { nicht ver- } \\
\text { gleichbar }\end{array}$ & $\begin{array}{l}\text { nicht ver- } \\
\text { gleichbar }\end{array}$ & & & DP nicht vergleichbar \\
\hline Bund 1953 & & ja & nein & & & BHE nein, DP ja \\
\hline BW 1953 & ja & ja & nein & & & unverändert \\
\hline SH 1954 & & ja & nein & & & BHE nein \\
\hline НB 1955 & ja & nein & nein & & & \\
\hline NRW 1956 & ja & & nein & & & $Z$ nein \\
\hline BW 1956 & ja & nein & nein & & & BHE ja \\
\hline BY 1957 & & ja & nein & & & BHE nein \\
\hline NDS 1957 & ja & ja & & & & BHE nein, DP ja \\
\hline BY 1958 & & ja & nein & & & BHE nein \\
\hline NDS 1959 & ja & & ja & & & BHE nein \\
\hline SL 1959 & & & & & & \\
\hline BW 1960 & & $\mathrm{ja}$ & nein & & & BHE nein \\
\hline BB 1990 & ja & & nein & nein & & \\
\hline НВ 1991 & nein & & nein & ja & & \\
\hline SAN 1994 & ja & & & nein & (nein) & \\
\hline HН 1901 & & ja & nein & & & Schill-Partei nein \\
\hline BE 1901 & ja & & & nein & (ja) & \\
\hline HE 2008 & nein & & & ja & unverändert & \\
\hline \multicolumn{7}{|c|}{$\begin{array}{l}\text { Anmerkung: Als Wahlerfolg wird gerechnet, wenn eine Partei bei der nächsten Wahl einen prozentualen } \\
\text { Zugewinn zu verzeichnen hat. } \\
\text { Quelle: Eigene Zusammen- und Darstellung, vgl. auch Aufsätze im Sammelband Uwe Jun / Melanie } \\
\text { Haas / Oskar Niedermayer (Hrsg.), Parteien und Parteiensysteme in den deutschen Ländern, Wiesbaden } \\
2009 .\end{array}$} \\
\hline
\end{tabular}

\begin{tabular}{|l|c|c|c|c|}
\hline \multicolumn{2}{|c|}{ Tabelle 5: Erfolg der Parteien nach komplexen Koalitionen } \\
\hline \multirow{2}{*}{} & \multicolumn{3}{|c|}{ Gesamt } & \multicolumn{2}{c|}{ Nach 1990 } \\
\cline { 2 - 5 } & Verbessert & Verschlechtert & Verbessert & Verschlechtert \\
\hline SPD & 11 & 2 & 3 & 2 \\
CDU/CSU & 11 & 2 & 1 & 0 \\
FDP & 1 & 15 & 0 & 3 \\
Grüne & 2 & 3 & 2 & 3 \\
PDS/Linke & 1 & 1 & 1 & 1 \\
BHE & 1 & 9 & & \\
DP & 2 & 2 & & \\
Zentrum & 0 & 2 & 0 & \\
Schill-Partei & 0 & 1 & \\
\hline Quelle: Eigene Darstellung, siehe auch Tabelle 4. \\
\hline
\end{tabular}


Scheitern der Koalitionsbemühungen in Schleswig-Holstein und Berlin vier beziehungsweise fünf Jahre später folgten, ebenfalls deutlich zulegten, so ist unter dem Gesichtspunkt parlamentarischer Teilhabe die Bilanz für die Grünen eher günstig.

\section{Fazit: Die Großen profitieren, die Kleinen haben Grund zur Sorge}

Komplexe Koalitionen sind aus Sicht von SPD und Union sehr ertragreiche Konstellationen. Sie umgingen damit nicht nur die ungeliebte Alternative Große Koalition, sondern profitierten von den komplexen Koalitionen. Meistens blieben beide Parteien auch nach deren Ende in der Regierung. Sprach der Wähler noch sein Urteil, wurden beide fast durchgängig dafür belohnt. Möglicherweise spielt dabei die Ressource Ministerpräsident eine Rolle. SPD und Union können aus der Regierung, gar aus der Regierungsführung heraus, sehr erfolgreich auch komplexe Koalitionen beenden oder zu Ende führen. Beide Parteien sind also in Bezug auf ihren Erfahrungsschatz prinzipiell gut für das Fünfparteiensystem gewappnet und brauchen keinerlei Scheu vor schwierigen und fragilen Bündnissen zu haben. Selbst im Falle des Scheiterns hat der Wähler hinterher die Arbeit der führenden Regierungspartei honoriert und ihren Verbleib in der Regierung gesichert.

Anders stellt sich die Lage für die FDP und die Grünen dar. Hatte die FDP in den 1950er Jahren ihre strategisch günstige Rolle im Parteiensystem nutzbringend für eine weitere Regierungsbeteiligung eingesetzt, so misslang ihr dies nach 1990 gründlich. Bei Wahlen hat die FDP stets Einbußen hinnehmen müssen, wenn sie sich einer komplexen Koalition anschloss. Die Grünen hingegen sicherten sich zwar nach Ende einer komplexen Koalition nie die anschließende Regierungsbeteiligung, konnten aber teilweise recht erfolgreich in nachfolgenden Wahlen abschneiden. Bei der PDS/Linken stellt sich die Bilanz sehr gemischt dar, so dass dort ein abschließendes Urteil, auch vor dem Hintergrund der einbezogenen Tolerierungen, schwierig ist.

Indes wird deutlich, warum gerade die kleineren Parteien sich schwertun, komplexe Koalitionen einzugehen. Sie müssen befürchten, in solchen Konstellationen Nachteile zu erleiden. Streben die großen Parteien also komplexe Koalitionen an, werden sie auf lange Sicht genau diese Nöte der kleineren Parteien in den Blick nehmen müssen.

\section{Der Aufstieg der Linkspartei - oder was passiert, wenn Loyalität schwindet}

Volquart Stoy und Josef Schmid

Mit der Entstehung der Linkspartei und ihrem wohl dauerhaften Einzug in den Bundestag hat sich die Transformation zu einem Fünfparteiensystem in Deutschland endgültig vollzogen. Das Wahlergebnis von 8,7 Prozent bei der Bundestagswahl 2005 bedeutete für die Linke nicht nur Rang vier in der Parteienhierarchie, sondern auch eine Verdoppelung ihres Stimmanteils im Vergleich zur Wahl von 2002 (damals noch als PDS). Bei der Wahl 2009 